\title{
CALCULATION OF SMALL DISTANCES*
}

\author{
By C. F. RICHTER
}

ABstract

TABLES are presented which facilitate the calculation of small distances between points on the earth's surface whose latitudes and longitudes are given, for mean latitudes from $30^{\circ}$ to $40^{\circ}$. The error does not exceed one-tenth of a kilometer in 500 kilometers, and may be reduced by applying specified corrections.

IN woRk relating to earthquakes local to an area under intensive seismological and geological investigation, epicenters are usually placed to within a few kilometers. Times of seismic waves are determined to the tenth of a second. Distances from stations to epicenters assumed for trial are required to the nearest kilometer; and it is desirable that the method used should be capable of furnishing such distances to the tenth of a kilometer, in order to avoid rounding-off errors during the calculations.

The last requirement usually causes trouble. To read distances of the order of 300 kilometers from a map, with an accuracy of $0.1 \mathrm{~km}$., means working on an awkwardly large scale, and checking the measurements with such care that they become very time-consuming. Computation from the coördinates of the stations and epicenter is much more desirable, provided tables and other material are available for carrying through the process with reasonable speed.

The following discussion contains no new contribution, but presents wellknown material in the form which has been found most convenient at Pasadena, for use with a computing machine.

Distances up to about $5^{\circ}(555 \mathrm{~km}$.) are given with an error not exceeding 0.1 $\mathrm{km}$. by a formula of the simple form

$$
\Delta^{2}=\Delta x^{2}+\Delta y^{2} ; \quad \Delta x=A \Delta \lambda, \quad \Delta y=B \Delta \Phi
$$

Here $\Delta$ is the required distance in kilometers, $\Delta \lambda$ and $\Delta \Phi$ are differences in longitude and latitude between the two given points (epicenter and station), and $A$ and $B$ are conversion factors from circular measure to kilometers. If $\Delta \lambda$ and $\Delta \Phi$ are in minutes of longitude and latitude, then sufficient accuracy is provided by taking $A$ and $B$ as the lengths in kilometers of one minute of arc of the parallel and meridian, respectively, taken at the mean latitude between the two points.

This method is equivalent to that known in navigation as "middle latitude sailing." It is a close approximation to that given by Wiechert (1925). His method, involving tables for finding factors equivalent to $A$ and $B$, is reasonably rapid in practice, especially after the user is familiar with it. The definition

* Manuscript received for publication January 20, 1943. 
here given for the factors $A$ and $B$ leads to the preparation of simple tables for much more rapid computation.

The Smithsonian Geographical Tables (Woodward, 1894) contain data from which table 1 is excerpted and derived.

For the accuracy required, five significant figures are usually sufficient. It is necessary then to interpolate the kilometer equivalents given in table 1 to apply to the mean latitude between the two points considered. For the factor $B$, applying to differences in latitude, this interpolation is simple, since the

TABLE 1

Lengths of Terrestrial Arcs, in Kilometers (Clarke Spheroid)

\begin{tabular}{|c|c|c|c|c|c|c|}
\hline \multirow{2}{*}{$\begin{array}{l}\text { Mean } \\
\text { latitude } \\
(\Phi)\end{array}$} & \multicolumn{2}{|c|}{ Arc of meridian, $B$} & \multicolumn{2}{|c|}{ Arc of parallel, $A$} & \multicolumn{2}{|c|}{$A / \cos \Phi$} \\
\hline & Degree & Minute & Degree & Minute & Degree & Minute \\
\hline $30^{\circ}$ & 110.8497 & 1.847495 & 96.4893 & 1.608155 & 111.4162 & 1.856937 \\
\hline 31 & 110.8669 & 1.847781 & 95.5073 & 1.591788 & 111.4220 & 1.857033 \\
\hline 32 & 110.8844 & 1.848073 & 94.4962 & 1.574937 & 111.4279 & 1.857132 \\
\hline 33 & 110.9023 & 1.848372 & 93.4563 & 1.557605 & 111.4339 & 1.857231 \\
\hline 34 & 110.9204 & 1.848673 & 92.3879 & 1.539798 & 111.4399 & 1.857331 \\
\hline 35 & 110.9388 & 1.848980 & 91.2913 & 1.521522 & 111.4461 & 1.857435 \\
\hline 36 & 110.9574 & 1.849290 & 90.1668 & 1.502780 & 111.4523 & 1.857538 \\
\hline 37 & 110.9763 & 1.849605 & 89.0148 & 1.483580 & 111.4586 & 1. 857643 \\
\hline 38 & 110.9953 & 1.849922 & 87.8356 & 1.463927 & 111.4650 & 1.857750 \\
\hline 39 & 111.0145 & 1. 850242 & 86.6296 & 1.443827 & 111.4715 & 1.857858 \\
\hline 40 & 111.0339 & 1. 850562 & 85.3970 & 1.423283 & 111.4779 & 1. 857965 \\
\hline
\end{tabular}

tabular differences in the second column of table 1 are very regular. There results the working table which is given as table 2 .

The factor $A$, applying to differences in longitude, is less simple. The differences in the third and fourth columns of table 1 are not smooth enough for easy interpolation. Each of these figures has accordingly been divided by the cosine of the corresponding latitude, which gives the quotients in the fifth and sixth columns. The differences in these are now regular enough for simple interpolation; this was performed for each $10^{\prime}$ of latitude, the interpolated values multiplied by their corresponding cosines, and a final interpolation performed to give values of $A$ for each minute of latitude. The final working table is given as table 3 .

Using tables 2 and 3 , with the aid of a computing machine and a good table of squares, routine calculation of distances is wery rapid and accurate. With the machine, it is more convenient to form the sums of squares directly instead of referring to the table of squares, which is used only in finding $\Delta$ at the last step. If no machine is available, the multiplications by $A$ and $B$ are not par- 
ticularly onerous; the squarings will then be performed with the aid of the table of squares. The following is an example.

Station, Pasadena, $34^{\circ} 08^{\prime} .9 \mathrm{~N}, 118^{\circ} 10^{\prime} .3 \mathrm{~W}$

Trial epicenter, $32^{\circ} 00^{\prime} \mathrm{N}, 119^{\circ} 00^{\prime} \mathrm{W}$

$$
\Delta \Phi=128^{\prime} .9 \quad \Delta \lambda=49^{\prime}: 7
$$

Mean latitude $\Phi=33^{\circ} 04 ! 45$

From table $2, B=1.8484$

From table $3, A=1.5562$

Hence $\Delta x=77.3 \mathrm{~km} ., \Delta y=238.3 \mathrm{~km}$.

$\Delta=\sqrt{77.3^{2}+238.3^{2}}=250.5 \mathrm{~km}$.

TABLE 2

CoEfFicients $B$

(Lengths in kilometers of $\mathbf{1}^{\prime}$ of the meridian)

\begin{tabular}{|c|c|c|c|c|c|c|c|}
\hline \multicolumn{3}{|c|}{ Mean latitude $\Phi$} & $B$ & \multicolumn{3}{|c|}{ Mean latitude $\Phi$} & $B$ \\
\hline \multicolumn{3}{|c|}{$29^{\circ} 51^{\prime}$ to $30^{\circ} 11^{\prime}$} & 1.8475 & $35^{\circ}$ & $14^{\prime}$ to $35^{\circ}$ & $33^{\prime}$ & 1.8491 \\
\hline \multirow[t]{3}{*}{30} & 12 to & 32 & 76 & & 34 to & 52 & 92 \\
\hline & 33 to & 53 & 77 & 35 & 53 to 36 & 11 & 93 \\
\hline & 54 to 31 & 14 & 78 & 36 & 12 to & 30 & 94 \\
\hline \multirow[t]{3}{*}{31} & 15 to & 34 & 79 & & 31 to & 49 & 95 \\
\hline & 35 to & 54 & 80 & & 50 to 37 & 08 & 96 \\
\hline & 55 to 32 & 14 & 81 & 37 & 09 to 2 & 27 & 97 \\
\hline \multirow[t]{3}{*}{32} & 15 to & 35 & 82 & & 28 to. & 46 & 98 \\
\hline & 36 to & 55 & 83 & & 47 to $38 c$ & 06 & 1.8499 \\
\hline & 56 to 33 & 15 & 84 & 38 & 07 to 2 & 24 & 1.8500 \\
\hline \multirow{3}{*}{33} & 16 to & 35 & 85 & & 25 to & 43 & 01 \\
\hline & 36 to & 55 & 86 & & 44 to 39 & 01 & 02 \\
\hline & 56 to 34 & 15 & 87 & 39 & 02 to 2 & 20 & 03 \\
\hline \multirow[t]{3}{*}{34} & 16 to & 34 & 88 & & 21 to & 39 & 04 \\
\hline & 35 to & 54 & 89 & & 40 to & 57 & 05 \\
\hline & 55 to 35 & 13 & 1.8490 & & 58 to 40 & 16 & 1.8506 \\
\hline
\end{tabular}

It remains to discuss the errors of the method. These fall into three classes: (1) errors due to the curvature of the earth considered as a sphere, (2) errors due to the departure of the Clarke spheroid (on which the geographical tables used are based) from a sphere, and (3) errors due to departure of the Clarke spheroid from the true figure of the earth, or from presumably better approximations such as the International Ellipsoid.

Since errors of the first class vanish when the two points are on the same meridian ( $\Delta \lambda=0$ ), while those of the other classes are then at maximum, a direct comparison is of interest. The following figures are typical:

Length of arc of the meridian between latitudes $31^{\circ} 30^{\prime}$ and $36^{\circ} 30^{\prime}$ :

1) multiplying by 300 the length given for $1^{\prime}$ of the meridian at $34^{\circ}$ in table 2, $554.61 \mathrm{~km}$. 
TABLE 3

Comfficients $A$ for Given Mean Latitude $\Phi$ (Lengths in kilometers of $1^{\prime}$ of the parallel)

\begin{tabular}{|c|c|c|c|c|c|c|c|c|c|c|}
\hline & $30^{\circ}$ & $31^{\circ}$ & $32^{\circ}$ & $33^{\circ}$ & $34^{\circ}$ & $35^{\circ}$ & $36^{\circ}$ & $37^{\circ}$ & $38^{\circ}$ & $39^{\circ}$ \\
\hline $00^{\prime}$ & 1. 6082 & 5918 & 1.5749 & 1.5576 & 1.5398 & 1.5215 & 1.5028 & 1.4836 & 1.4639 & 1.4438 \\
\hline & & 15 & 47 & 73 & 95 & 12 & 25 & & 36 & 35 \\
\hline & 76 & 12 & 44 & 70 & 92 & 09 & 21 & & 33 & 32 \\
\hline 0 & 74 & 10 & 41 & 67 & 89 & 06 & 18 & 26 & 29 & 28 \\
\hline & 71 & 07 & 38 & 64 & 86 & 03 & 15 & 23 & 26 & 25 \\
\hline & 1.6068 & 1.5904 & 1.5735 & 1.5561 & 1.5383 & 1.5200 & 1.5012 & 1.4820 & 1.4623 & 1.4421 \\
\hline & 65 & 1.5901 & 32 & 58 & 80 & 1.5197 & 09 & 16 & 19 & 18 \\
\hline 0 & 63 & 1.5898 & 29 & 56 & 77 & 94 & 06 & 13 & 16 & 15 \\
\hline 08 & 60 & 96 & 27 & - 53 & 74 & 90 & 1.5002 & 10 & 13 & 11 \\
\hline 09 & 57 & 93 & 24 & 50 & 71 & 87 & 1. 4999 & 07 & 09 & 08 \\
\hline 10 & 1.6055 & 1.5890 & 1.5721 & 1.5547 & 1.5368 & 1.5184 & 1.4996 & 1.4803 & 1.4606 & 1.4404 \\
\hline 1 & & 87 & 18 & 44 & 65 & 81 & 93 & 1.4800 & 1.4603 & 1.4401 \\
\hline 1 & 49 & 85 & 15 & 41 & 62 & 78 & 90 & 1.4797 & 1.4599 & 1.4398 \\
\hline 13 & 46 & 82 & 12 & 38 & 59 & 75 & 87 & 94 & 96 & 94 \\
\hline 1 & 44 & 79 & 09 & 35 & 56 & 72 & 83 & 90 & 93 & 91 \\
\hline 1 & 1. 6041 & 1.5876 & 1.5706 & 1.5532 & 1.5353 & 1.5169 & 1.4980 & 1.4787 & 1.4589 & 1.4387 \\
\hline 16 & 38 & 73 & 04 & 29 & 50 & 66 & 77 & 84 & 86 & 84 \\
\hline 1 & 36 & 71 & 1.5701 & 26 & 47 & 63 & 74 & 81 & 83 & 81 \\
\hline & 33 & 68 & 1.5698 & 23 & 44 & 60 & 71 & 78 & 79 & 77 \\
\hline 1 & 30 & 65 & 95 & 20 & 41 & 56 & 67 & 74 & 76 & 74 \\
\hline 2 & 1.6028 & 1.5862 & 1.5692 & 1.5517 & 1.5338 & 1.5153 & 1.4964 & 1.4771 & 1.4573 & 1.4370 \\
\hline 2 & 25 & 59 & 89 & 14 & 35 & 50 & 61 & 68 & 69 & 67 \\
\hline 2 & 22 & 57 & 86 & 11 & 32 & 47 & 58 & 64 & 66 & 63 \\
\hline 2 & 19 & 54 & 83 & 08 & 28 & 44 & 55 & 61 & 63 & 60 \\
\hline 2 & 17 & 51 & 81 & 05 & 25 & 41 & 52 & 58 & 59 & 56 \\
\hline 2 & 1.6014 & 1.5848 & 1.5678 & 1.5502 & 1.5322 & 1.5138 & 1.4948 & 1.4754 & 1.4556 & 1.4353 \\
\hline 2 & 11 & 45 & 75 & 1. 5499 & 19 & 35 & 45 & 51 & 53 & 50 \\
\hline 2 & 08 & 43 & 72 & 96 & 16 & 31 & 42 & 48 & 49 & 46 \\
\hline & 06 & 4 & 69 & 93 & 13 & 28 & $3 !$ & 45 & 46 & 43 \\
\hline 2 & 03 & 37 & 66 & 91 & 10 & 25 & 36 & 41 & 43 & 40 \\
\hline$\overline{3}$ & 1.6000 & 1.5834 & 1.5663 & 1.5488 & 1.5307 & 1.5122 & 1.4932 & 1.4738 & 1. 4539 & 1.4336 \\
\hline 3 & 1.5998 & 31 & 60 & 85 & 04 & 19 & 29 & 35 & 36 & 33 \\
\hline 32 & 95 & 29 & 58 & 82 & 1.5301 & 16 & 26 & 32 & 33 & 29 \\
\hline 3 & 9 & 26 & 55 & 79 & 5298 & 13 & 2 & 2 & 29 & 26 \\
\hline 3 & 89 & 23 & 52 & 76 & 95 & 10 & 20 & 25 & 26 & 22 \\
\hline 35 & 1.5987 & 1.5820 & 1.5649 & 1.5473 & 1.5292 & 1.5106 & 1.4916 & 1.4722 & 1.4523 & 1.4319 \\
\hline 36 & 84 & 17 & 46 & 70 & 89 & 03 & 13 & 18 & 19 & 16 \\
\hline 37 & 8 & 15 & 43 & 67 & 86 & 1.5 & 10 & 1 & 16 & 12 \\
\hline 3 & 7 & 1. & 40 & 6 & 83 & 1.5097 & 0 & & 1 & 09 \\
\hline 3 & 76 & 09 & 37 & 61 & 80 & 94 & 04 & 09 & 09 & 05 \\
\hline 40 & 1.5973 & 1.5806 & 1.5634 & 1.5458 & 1.5277 & 1.5091 & 1.4900 & 1.4705 & 1.4506 & 1.4302 \\
\hline 41 & 70 & & 31 & 55 & 74 & 88 & 1.4897 & & & 1.4298 \\
\hline 4 & & 1.5800 & 29 & 5 & 7 & 8 & 94 & 1.4699 & .4499 & 95 \\
\hline 43 & 6 & 1.5798 & 26 & 48 & 67 & 81 & 91 & 95 & 96 & 92 \\
\hline 44 & 62 & 95 & 23 & 46 & 64 & 78 & 87 & 92 & 92 & 88 \\
\hline 45 & 1.5959 & 1.5792 & 1.5620 & 1.5443 & 1.5261 & 1.5075 & 1.4884 & 1.4689 & 1. 4489 & 1.4285 \\
\hline 46 & 57 & 89 & 17 & 40 & 58 & 72 & 81 & 8 & 86 & 81 \\
\hline 47 & & 8 & 14 & 3 & 5 & 6 & 78 & 8 & 82 & 78 \\
\hline 48 & 5 & 83 & 11 & 34 & 52 & 66 & 75 & 79 & 79 & 74 \\
\hline 49 & & 81 & 08 & 31 & 49 & 63 & 71 & 76 & 75 & 71 \\
\hline 50 & 1.5946 & 1.5778 & 1.5605 & 1.5428 & 1.5246 & 1. 5059 & 1.4868 & 1.4672 & 1.4472 & 1.4267 \\
\hline 51 & 43 & 75 & 1.5602 & 24 & 43 & 56 & 65 & 69 & 69 & 64 \\
\hline 52 & 40 & 7 & 1.5599 & 22 & 40 & 53 & 62 & 66 & 65 & 60 \\
\hline 53 & 37 & 69 & 97 & 19 & 37 & 50 & 58 & 6 & 62 & 57 \\
\hline 54 & 34 & 66 & & 16 & 34 & & 55 & 59 & 59 & 54 \\
\hline 55 & 1.5932 & 1.5764 & 1.5591 & 1.5413 & 1.5231 & 1.5044 & 1.4852 & 1.4656 & 1.4455 & 1.4250 \\
\hline 56 & 29 & 61 & 88 & 10 & 28 & 40 & 49 & 52 & & \\
\hline 57 & & & 85 & 07 & 24 & 37 & 45 & 49 & 48 & \\
\hline & & & 82 & 04 & 21 & 34 & 42 & 46 & 45 & 40 \\
\hline 5 & & 52 & 79 & 1.5401 & 18 & 31 & 39 & 43 & 42 & 36 \\
\hline 6 & 1. 5918 & 1.5749 & 1.5576 & 1.5398 & 1.5215 & 1.5028 & 1.4836 & 1.4639 & 1.4438 & 1.4233 \\
\hline
\end{tabular}


2) multiplying by 5 the length given for $1^{\circ}$ of the meridian at $34^{\circ}$ in table 1 , $554.6020 \mathrm{~km}$.

3) by summing the lengths given at $32^{\circ}, 33^{\circ}, 34^{\circ}, 35^{\circ}$, and $36^{\circ}$ in table 1 , $554.6033 \mathrm{~km}$.

4) from tables based on the International Ellipsoid (Lambert and Swick, $1935), 554.6267 \mathrm{~km}$.

The third result is the correct length for the Clarke Spheroid. The first and second computations differ from this by the deviation of the spheroid from a sphere, plus rounding-off errors. This deviation is seen to be actually less than that between lengths computed on the two spheroids, and amounts to less than $0.01 \mathrm{~km}$., or less than ten meters.

The deviation between distances computed from formula (1) and distances over the sphere is increased when the end points also differ in longitude, while the effect of the ellipticity of the earth becomes less. Hence, in estimating errors of the method it will be sufficient to determine the errors when applied to points at the same latitude and longitude on a true sphere of nearly the size of the earth-conveniently, a sphere of $40,000 \mathrm{~km}$. circumference. The theoretical foundation is as follows.

Exact angular distances over the sphere are given by

$$
\cos \Delta=\cos \Phi_{1} \cos \Phi_{2} \cos \Delta \lambda+\sin \Phi_{1} \sin \Phi_{2}
$$

where $\Phi_{1}$ and $\Phi_{2}$ are the latitudes of the two given points, the longitudes of which differ by $\Delta \lambda$. If we put $\Phi_{1}-\Phi_{2}=\Delta \Phi$ and $\Phi_{1}+\Phi_{2}=2 \Phi$, and suppose $\Delta \Phi$ and $\Delta \lambda$ to be small compared to one radian, then we find

$$
\cos \Delta=1-\frac{\Delta \Phi^{2}}{2}-\cos ^{2} \Phi \frac{\Delta \lambda^{2}}{2}+\frac{\Delta \Phi^{4}}{24}+\frac{\Delta \Phi^{2} \Delta \lambda^{2}}{8}+\cos ^{2} \Phi \frac{\Delta \Phi^{4}}{24}+\cdots
$$

By applying the expansion of $\cos \Delta$ and using the method of undetermined coefficients it is easy to derive

$\Delta^{2}=\Delta \Phi^{2}+\cos ^{2} \Phi \Delta \lambda^{2}+\left(-\frac{1}{4}+\frac{\cos ^{2} \Phi}{6}\right) \Delta \Phi^{2} \Delta \lambda^{2}-\frac{\sin ^{2} \Phi \cos ^{2} \Phi}{12} \Delta \lambda^{2}+\cdots$

Writing $\Delta_{0}^{2}=\Delta \Phi^{2}+\cos ^{2} \Phi \Delta \lambda^{2}$, there results

$$
\Delta=\Delta_{0}-\frac{1}{24} \sin ^{2} \Phi \Delta_{0} \Delta \lambda^{2}-\frac{1}{24}\left(1+\sin ^{2} \Phi\right) \frac{\Delta \Phi^{2} \Delta \lambda^{2}}{\Delta_{0}}+\cdots
$$

$\Delta_{0}$ is the value of $\Delta$ given by the method of this paper. The following terms give the correction, including small quantities of the third order. If $\Delta \Phi, \Delta \lambda$, and $\Delta_{0}$ 
are near $6^{\circ}$ they are of the order of one-tenth of a radian, and the correction terms given above are of the order of one-thousandth of a radian divided by 24 , or about one-fourth of a kilometer.

In computing tables showing these errors it has been desirable to have $\Delta \Phi$ and $\Delta \lambda$ expressed in integral numbers of degrees, and $\Delta$, with the corrections, in kilometers. If $\Delta \Phi=m$ degrees and $\Delta \lambda=n$ degrees, then

$$
\Delta=\Delta_{0}-0.00140 \frac{n^{2}}{\sqrt{m^{2}+\cos ^{2} \Phi}}\left[\left(1+2 \sin ^{2} \Phi\right) m^{2}+\sin ^{2} \Phi \cos ^{2} \Phi m^{2}\right]
$$

for a sphere of $40,000 \mathrm{~km}$. circumference. The exact value of the numerical coefficient in the correction term is $\pi^{2} / 6998.4$.

TABLE 4

Errors of Formola (1), in Kilometers

\begin{tabular}{|c|c|c|c|c|c|c|}
\hline \multicolumn{7}{|c|}{$\begin{array}{c}\text { a. Mean latitude } \Phi=30^{\circ} \\
\Delta \lambda\end{array}$} \\
\hline$\Delta \Phi$ & $1^{\circ}$ & $2^{\circ}$ & $3^{\circ}$ & $4^{\circ}$ & $5^{\circ}$ & $10^{\circ}$ \\
\hline $0^{\circ}$ & .0003 & .002 & .008 & .02 & .04 & .31 \\
\hline 1 & .002 & .006 & .01 & .03 & .05 & .33 \\
\hline 2 & .004 & .01 & .03 & .05 & .08 & .39 \\
\hline 3 & .006 & .02 & .05 & .08 & .12 & .50 \\
\hline 4 & .008 & .03 & .07 & .12 & .17 & .63 \\
\hline 5 & .01 & .04 & .09 & .15 & .22 & .79 \\
\hline 10 & .02 & .08 & .19 & .32 & .50 & 1.80 \\
\hline
\end{tabular}

b. Mean latitude $\Phi=45^{\circ}$

$\Delta \lambda$

\begin{tabular}{c|l|l|l|l|l|l}
\hline$\Delta \Phi$ & $1^{\circ}$ & $2^{\circ}$ & $3^{\circ}$ & $4^{\circ}$ & $5^{\circ}$ & $10^{\circ}$ \\
\hline $0^{\circ}$ & .0005 & .0004 & .01 & .03 & .06 & .50 \\
1 & .003 & .01 & .02 & .05 & .08 & .53 \\
2 & .005 & .02 & .05 & .08 & .12 & .63 \\
3 & .008 & .03 & .07 & .12 & .18 & .79 \\
4 & .01 & .04 & .10 & .17 & .25 & .99 \\
5 & .01 & .06 & .12 & .21 & .32 & 1.22 \\
10 & .03 & .11 & .25 & .44 & .69 & 2.59 \\
\hline
\end{tabular}

Tables $4 a$ and $4 b$ have been computed from formula (6). Table $4 a$ gives the value of the third-order terms for mean latitude $\Phi=30^{\circ}$; table $4 b$, for $\Phi=45^{\circ}$. Interpolation can be made very exact, if desired, by noting that when both $\Delta \Phi$ and $\Delta \lambda$ are multiplied by the same constant $k$, the errors as tabulated are 
multiplied by $k^{3}$. These positive numbers are errors of formula (1); to be applied as corrections they must be subtracted, in accordance with the negative sign in (6).

The errors near $45^{\circ}$ are rougly half again as large as those near $30^{\circ}$, and errors increase further at higher latitudes. The following are the errors in meters for $\Delta \Phi=\Delta \lambda=1^{\circ}$, at the indicated mean latitudes:

$\begin{array}{cccccccc}\Phi & 30^{\circ} & 35^{\circ} & 40^{\circ} & 45^{\circ} & 50^{\circ} & 55^{\circ} & 60^{\circ} \\ \text { Error } & 1.80 & 2.05 & 2.32 & 2.59 & 2.87 & 3.14 & 3.39 \text { meters }\end{array}$

To compare with table 4 , divide by 1000 .

While these corrections are computed for a sphere of $40,000 \mathrm{~km}$. circumference, so that $1^{\circ}$ of a great circle has the length $111.1111 \mathrm{~km}$., they will apply with their tabulated accuracy to the true earth and to the results from formula (1) and tables 2 and 3 .

The method of construction of tables 2 and 3 eliminates that part of the effect of the ellipticity of the earth which expresses itself in the difference between the ordinary, geodetic or geographical latitude (which is that used in the tables and on all ordinary maps), and the geocentric latitude. This effect must be considered in any attempt to make direct use of equation (2), since neglecting it will introduce comparatively large errors. As an example, suppose formula (1) and the tables used to find a distance between two points at latitudes $32^{\circ}$ and $36^{\circ}$, differing $4^{\circ}$ in longitude. The mean latitude $\Phi$ is then $34^{\circ}$. Tables 2 and 3 give $\Delta x=369.552$ and $\Delta y=443.688$, whence $\Delta=577.432$ kilometers. On the other hand, equation (2) with $\Phi_{1}=32^{\circ}, \Phi_{2}=36^{\circ}$, and $\Delta \lambda=4^{\circ}$, gives $\cos \Delta=0.9958928$, whence $\Delta=5^{\circ} 11^{\prime} 40^{\prime \prime} .9=5.19469^{\circ}$. Now the length of $1^{\circ}$ of a great circle at the mean latitude is that given in table 1 for $1^{\circ}$ of the meridian centering at $34^{\circ}$, namely, $110.9204 \mathrm{~km}$. Multiplying this by the last value for $\Delta$ expressed in degrees, we find $\Delta=576.197$. There is a discrepancy of $1.235 \mathrm{~km}$. between the two results.

The error originates in the use of geodetic latitudes in equation (2). The computed value of $\cos \Delta$, and of $\Delta$ derived from it, should refer to points at geocentric latitudes $32^{\circ}$ and $36^{\circ}$. The corresponding geodetic latitudes are $32^{\circ} 10^{\prime} 30^{\prime \prime}$ and $36^{\circ} 11^{\prime} 07^{\prime \prime}$. The arc of the meridian between these latitudes is found by interpolation in table 1 to be $444.814 \mathrm{~km}$., so that $1^{\circ}$ of the great circle is $111.203 \mathrm{~km}$. Multiplying this by 5.19469 gives $\Delta=577.665 \mathrm{~km}$., which is probably the best result obtainable by simple means. The values of $\Delta x$ and $\Delta y$ used in formula (1) also need correction, since the geodetic mean latitude $\Phi$ should now be taken as $34^{\circ} 10^{\prime} .8$, while the difference in latitude is not $240^{\prime}$ but 240 '.61. Tables 2 and 3, then, give $\Delta x=368.760 \mathrm{~km}$., $\Delta y=444.816 \mathrm{~km}$., whence $\Delta=577.793 \mathrm{~km}$. A more exact value of $\Delta x$, derived from table 1 , is $368.770 \mathrm{~km}$., while more exactly $\Delta y=444.814 \mathrm{~km}$. as found earlier. These 
give $\Delta=577.798 \mathrm{~km}$. as the best result using formula (1). The correction to this derived from formula (6) is $-0.0127 \mathrm{~km}$., giving a corrected result of $\Delta=577.671 \mathrm{~km}$. This differs from the result of (2) by $0.006 \mathrm{~km}$., which is due to slight rounding-off errors.

The quantities $\Delta x$ and $\Delta y$ are independently useful in least-square determinations of epicenter and origin time. Their quotient gives the tangent of the angle which measures the azimuth of the station at the epicenter.

Mr. John M. Nordquist has assisted by verifying and extending some of the numerical examples and formulas, and also with the computation of tables based on formula (6).

Balch Graduate School of the Geological Sciences

California Institute of Technology, Pasadena, Calmornia

CONTRIBUtion No. 351.

\section{REFERENCES}

LAMBert, W. D., and Swick, C. H. "Formulas and Tables for the Computation of Geodetic Positions on the International Ellipsoid," U. S. Coast and Geodetic Survey, Spec. Publ. No. 200 (1935).

WIECRERT, E. (1925). "Entfernungsberechnungen von Orten auf der Erde bei kleineren Abständen," Zeitschr. f. Geophysik, I, 176-191.

Woodward, R. S. (1894). Smithsonian Geographical Tables, Washington, Smithsonian Institution. (3d ed., 1918). 